\title{
Hydrodynamics features in cavitation devices under tangent medium input
}

\author{
Tetiana Vitenko ${ }^{1, *}$, Paweł Droździel ${ }^{2}$, Nazar Horodyskyi ${ }^{1}$ \\ ${ }^{1}$ Ivan Puluj TNTU, Faculty of Industrial Machinery Engineering, str. Ruska 56, 2-80 Teronpil, \\ vitenko@tntu.edu.ua,nazar_horodysky@ukr.net, Ukraine \\ ${ }^{2}$ Lublin University of Technology, Faculty of Mechanical Engineering, Nadbystrzycka 36 Street, \\ 20-618 Lublin,p.drozdziel@pollub.pl, a.rudawska@pollub.pl, Poland
}

\begin{abstract}
Theoretically analysed design features of the working section of a hydrodynamic cavitation device with swirling input. Using the numerical methods of SolidWorks software and the Flow simulation module, fluid flow modeling was performed to describe similar processes. The comparison of the results of visual and experimental studies has been carried out. Visual observations indicate that the life cycle of vapor-gas bubbles and cavities in the design under study can be divided into several periods, in particular the formation of vapor-gas bubbles, their growth, generation of cavities, their volume increase, transformation and destruction. The influence of input and output hydrodynamic and technological parameters of the flow and design features on the character of the cavitation area and the form of cavitation were studied. According to the results of theoretical and experimental research, rational mode of operation of the cavitation device is proposed.
\end{abstract}

Keywords: cavitation, hydrodynamics, bubbles, collapse, SolidWorks.

\section{Introduction}

The question of finding effective intensification methods for the technological processes of the chemical, food or pharmaceutical industries today does not lose its relevance. Particular attention is attracted to the dynamic effects arising from the development of bubble systems during cavitation. The basis of one of the concepts on which the explanation of the mechanism of cavitation influence on technological systems is based, is the theory of discrete-impulse energy input (DIEI) introduced into the apparatus. The references describe in detail general principles of DIEI, describe the energy and thermodynamic aspects and the main intensification mechanisms that can be initiated on the basis of this principle $[1,2]$. DIEI mechanisms conditionally can be divided into hard ones and soft ones. The former should be used to stimulate hydromechanical processes, and the latter to accelerate the processes of phase heat and mass transfer, or for the purpose of intensive mixing of multicomponent media, when the level of intensification of the process can be smoothly changed within certain limits. In practice, such conditions are achieved by ultrasonic influence on the liquid [3] or by creating special hydrodynamic conditions in the flow in propellant or rotary cavitation devices. The cavitation mechanism is also implemented in

\footnotetext{
* Corresponding author: vitenko@tntu.edu.ua

Reviewers: Vladimír Vrba, Dušan Štekláč
} 
rotary-pulsating homogenizers and in industrial apparatuses. The phenomenon of steam cavitation was studied under injection of superheated steam into a cold liquid [4].

Propellant cavitation devices using flow energy for the formation of a tear-off flow are effectively used in industry for the intensification of heat-mass transfer processes. The idea of their use is based on the cavitation effects, which are accompanied by the cold boiling of the liquid. The difference is that when increasing the relative flow velocity relative to the body up to the critical value, the pressure of the flow decreases up to the saturation vapor pressure. In this case, the liquid boils, forming cavitation gas-vapor bubbles of various sizes and caverns, which get into the area of high pressure, where they collapse (close up, condense). It was investigated that at these points, local pressures and temperatures are significantly increased [1-3]. If the bubbles collapse directly at the surface or the disperse particle, then the kinetic energy of the radial motion of the fluid is converted into the mechanical energy of a liquid cumulative jet that has a very high velocity and moves to this surface. This mechanism is described in the authors' studies [5].

Significant interest is caused by dynamic effects of varying intensity, which are determined by the stage of cavitation bubbles collapse and identify the effectiveness of the cavitation activity on the process media. The life cycle of cavitation bubbles was studied by the authors [5]. The method of high-speed filming shows the development of a spherical bubble from its formation to destruction. The theoretical analysis of these experiments was carried out using Rayleigh equation in the Plesset's work. Subsequent studies of the dynamics of gas-vapor bubbles took into account the kinetics of heat and mass transfer processes. The complex of studies and their generalization was carried out in papers $[6,7]$, which set out universal mathematical models that, from single thermodynamic positions, adequately describe the dynamics of individual bubbles and bubble ensembles under cavitation conditions.

Numerical simulation methods are widely used for planning and analyzing the results of experiments, research and study of such processes. Numerical models of cavitation are divided into three groups: Conditional, Mass, Euler. In [8], the author analyzed the "Conditional" models, which are divided into two types, depending on the hydrodynamic conditions that determine the cavitation number, the saturation vapor pressure, and the local flow velocity. Mass transfer in this class of models is not considered. The advantage of such models is the speed of obtaining a solution, but there is a certain difficulty in indicating the cavitation number. Such models are usually used for initial engineering calculations, under the condition that the cavitation occurs under the indicated cavitation number. At the same time, this does not abolish the need for an experiment to determine the exact value of the cavitation number.

Euler model allows to calculate steam and liquid characteristics. The possibility of simulation hydrodynamic cavitation under conditions of slow and high-speed fluid flows, developed cavitation, non-stationary currents are the advantages of this model. The disadvantages of Euler model should include the duration of the calculation. These models are used for simulation cavitation in hydropower plants and high-speed centrifugal pumps. It is also possible to model cavitation in vortex tubes with the connection of thermodynamic ratios. Models of this type are present in ANSYS Fluent and ANSYS CFX [9].

It should be noted that the cavitation processes described in accordance with the laws of mathematical simulation are practically impossible to describe by the behaviour of individual bubbles or even their groups that define certain parameters. On the other hand, it is possible to describe some of the processes in a particular situation using the dimension theory and general parameters such as geometry, external pressure, temperature, saturation vapor pressure at a certain temperature, properties of the working fluid (viscosity, density, compressibility). In this case, the simulation of the cavitation flow is based on the fundamental laws of fluid/gas mechanics, in particular on the equations of continuity, 
momentum equations, and the law of variation of turbulent viscosity from the velocity (turbulence model). In this context, it should be noted that the above mentioned theoretical and experimental studies of cavitation flows hydrodynamics features, depending on the design of the equipment, have allowed to solve an important task of forecasting its efficiency and prospects for further application.

\section{Description of the main research material}

In this paper, the simulation was conducted to study cavitational areas characteristics and the choice of rational regime parameters of the system's influence on the proposed design. The effect of input and output hydrodynamic flow parameters and geometric design features on the character of the cavitation area was studied. The work of the system was considered under the condition of the previous flow swirling, which provides acceleration of the flow at the inlet, additional fluid pulsations and increase the intensity of the impact on the technological environment. The photo of the working area "cone-working body" of the experimental installation is shown in Fig. 1.

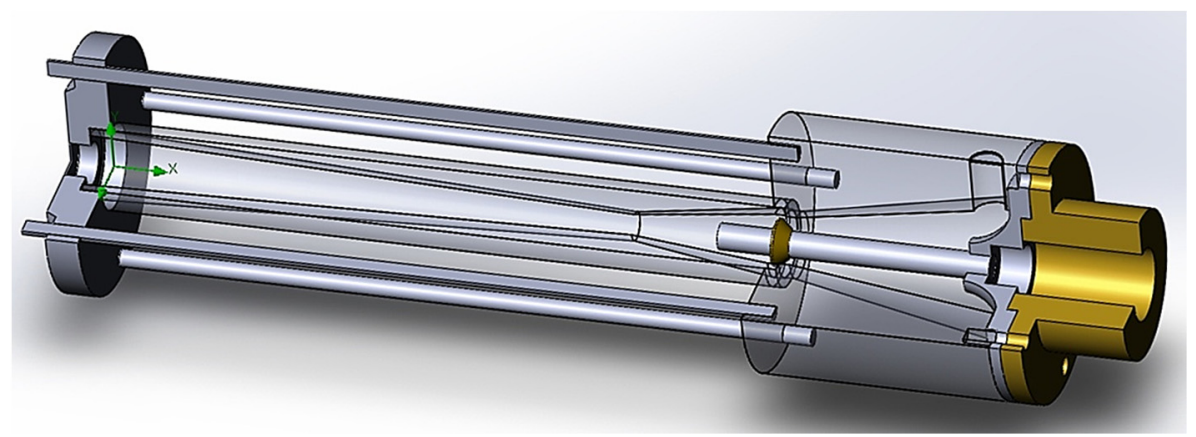

Fig. 1. Installation section in the cone area

Due to the rotation in the inlet pipe, the fluid flow becomes much more mobile (moving) along the central axis and acquires increased kinetic energy. Excessive pressure created in the inlet pipework provides fluid transfer through the narrowing and expansion part of the system. An increase in the fluid velocity in the narrow section of the Venturi tube causes a drop in local static pressure. The gradual increase in the flow of liquid leads to a reduction in pressure to a critical, equal to or close to the pressure of saturated vapor of the liquid, and the emergence of cavitation phenomena, which can be explained by the processes occurring around and within the vapor-gas phase.

Visual observations have shown that life cycle of vapor-gas bubbles and cavities in such a design can be conditionally divided into several periods. Consistent formation of vapor-gas bubbles, their growth, generation of caverns, increase of their volume, transformation and destruction. It was established that for the structure under study, the appearance of cavitation and the appearance of separate cavitation bubbles occurs under $\delta=\delta_{\text {crit }}=2 \mathrm{~mm}$.

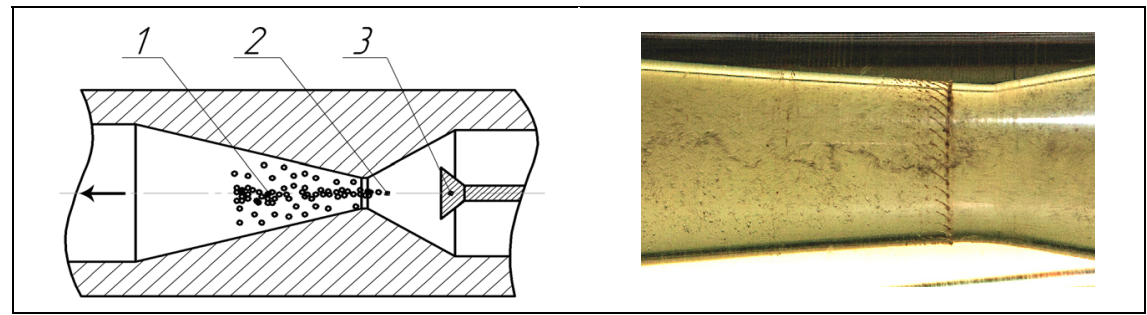

Fig. 2. Emergence of separate cavitation bubbles 
At the corresponding value of pressure $\mathrm{P}_{2}$ at the output of the tube, occurs the formation and growth of the cavity over the obstacle 3, the length of which increases over time, it extends and takes channel 2 and goes out to the diffuser 1 (Fig. 3).

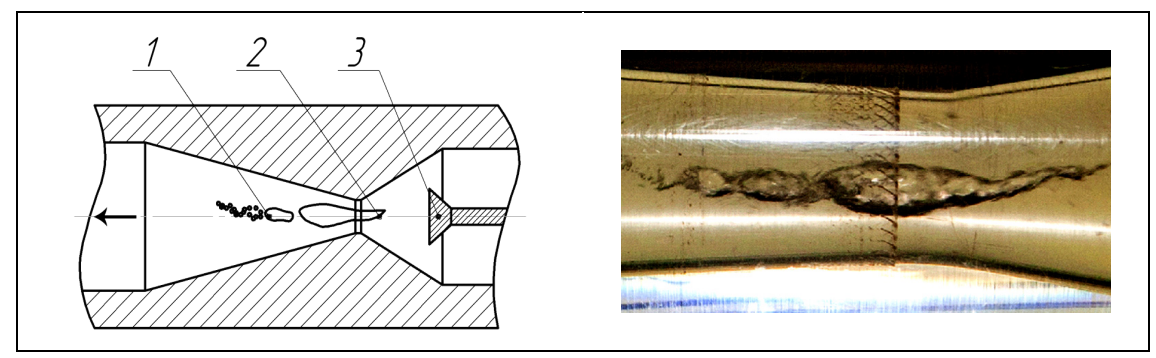

Fig. 3. Formation of the cavity

After one cavity separates into several ones, the new one starts to emerge on its place; the previous one goes down the stream and collapses. As the studies have shown, the process of cavity`s part separating takes place strictly periodically, that is, with a stable frequency $f$. There is a formation of periodically tearing-off flow, the distinctive feature of which is the stability of the separation frequency and collapse (in the stream, and not on the wall) of caverns (Fig. 4).

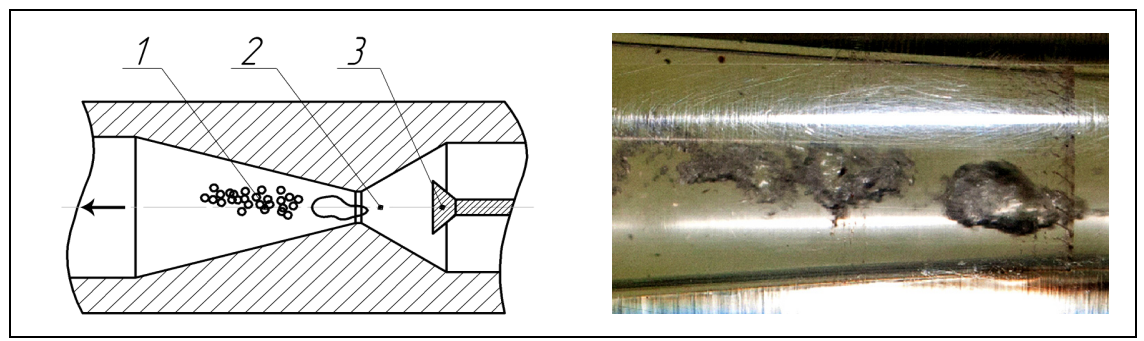

Fig. 4. Partition and separation of the cavity

Visual observations also showed that the cavitational cavity begins to form at the critical value of the cavitation number $\sigma=1.6$. At the beginning, cavitation is of the bubble type, with a decrease of $\sigma$ up to 1 is observed film flow, the size of the cavity is $0.005-0.007 \mathrm{~m}$. With a decrease of $\sigma$ up to 0.9 , the type of cavitation changes, and the average length of the cavity increases up to $0.015-0.02 \mathrm{~m}$. Cavities of small size are destroyed and form bubbles (Fig. 5). The destruction of the cavity is also facilitated by the local energy release, the formation of local pressure gradients that occur as a result of collapse of cavitation bubbles.

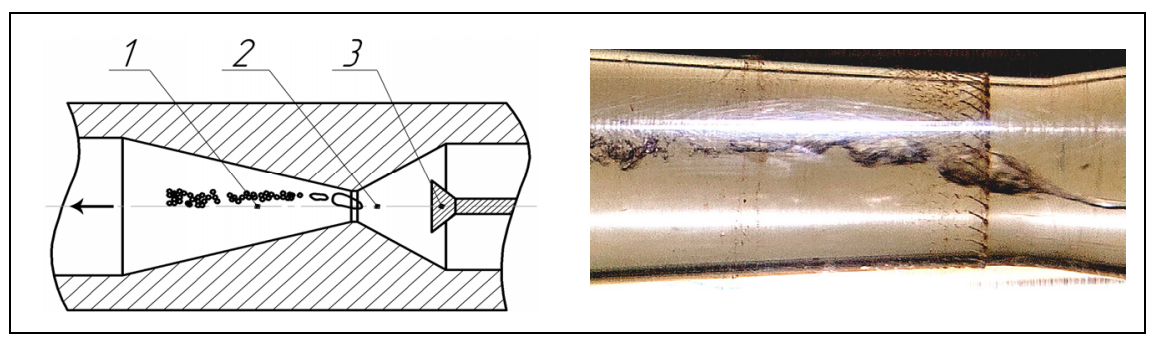

Fig. 5. Evolution of small size cavities 
To study the possibility of using numerical methods to describe such processes, simulation was performed in the SolidWorks software complex using the Flow simulation module, and the results were compared with visual and experimental studies. Changes in pressure $\mathrm{P}$ within the area behind the diffuser are shown in Fig. 6. According to the obtained results under the input pressure $\mathrm{P}_{1}=0.2 \mathrm{MPa}$, the pressure at the outlet from the pipeline $\mathrm{P}_{2}$ is 0.5 from $\mathrm{P}_{1}$ $(0.1 \mathrm{MPa})$. The pressure discontinuity within the area of the diffuser and behind it are Pmax $=1.2-1.3 \mathrm{P}_{1}$, i.e. $0.24-0.26 \mathrm{MPa}, \mathrm{Pmin}=0.1 \mathrm{P}_{2}$, therefore, it is about $0.03 \mathrm{MPa}$. According to the obtained calculations, the pressure in the volume of liquid $\mathrm{P}$ falls in the diffuser from $0.2 \mathrm{MPa}$ up to $0.17 \mathrm{MPa}$ in front of the cone, then decreases up to $0.12 \mathrm{MPa}$ at the entrance to the cylindrical area. Next comes the second wave of pressure drop from $0.12 \mathrm{MPa}$ to $0.026 \mathrm{MPa}$, which is confirmed by the result of the experiment. In general, the static pressure along the flow line in the working chamber varies: the pressure on the periphery is higher than along the axis (Fig. 6), as the vortex flow has centrifugal forces directed from the center to the outer wall of the tube. Then the pressure increases from 0.02 up to $0.1 \mathrm{MPa}$.

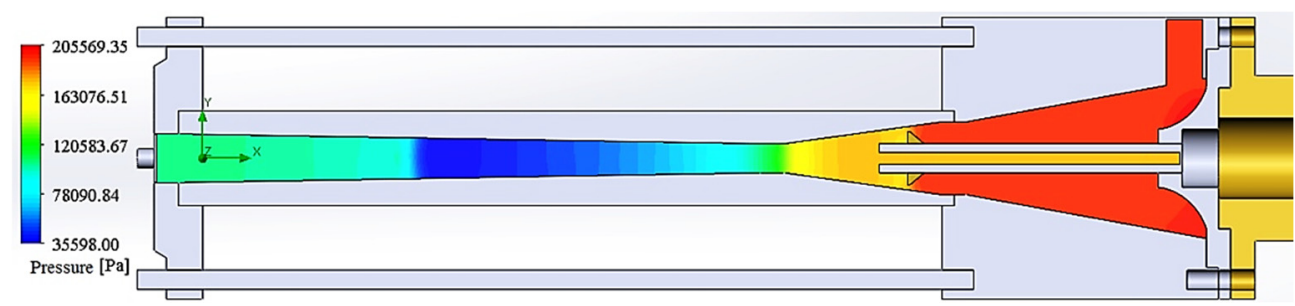

Fig. 6. Pressure distribution $\left(\delta_{\text {crit }}=2 \mathrm{~mm}\right)$ pressure at the input $0.2 \mathrm{MPa}$

Similar calculations are performed under other input pressures within the limits $\mathrm{P}_{1}=0.3$ $0.6 \mathrm{MPa}$ at different values of $\delta_{\mathrm{\kappa}} \mathrm{p}$. Adequate results are obtained according to the experiment measured better than $10 \%$.

In Fig. 7 is shown the distribution of vapor volume concentration in the working channel for different modes $\left(\mathrm{P}_{1}=0.2\right.$ and $\left.\mathrm{P}_{1}=0.4 \mathrm{MPa}, \delta_{\text {crit }}=2 \mathrm{~mm}\right)$. The smallest cavitation area (Fig. 7 (b)) corresponds to the input pressure $\mathrm{P}_{1}=0.4 \mathrm{MPa}$.

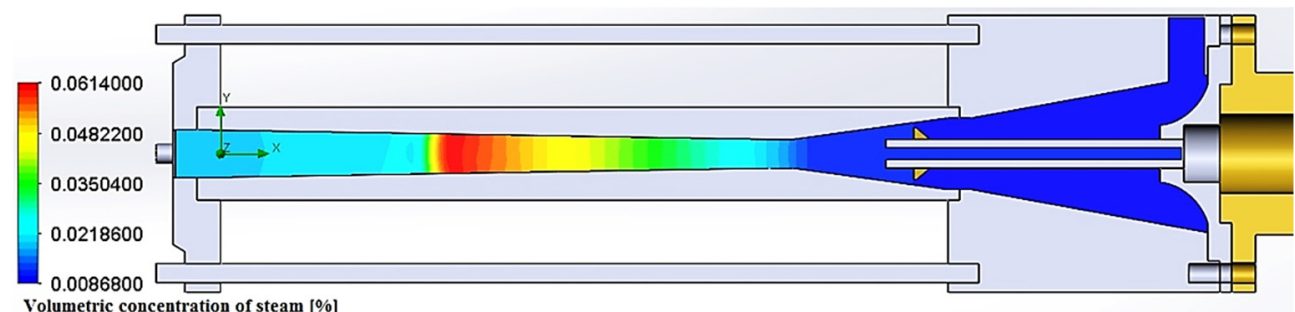

a

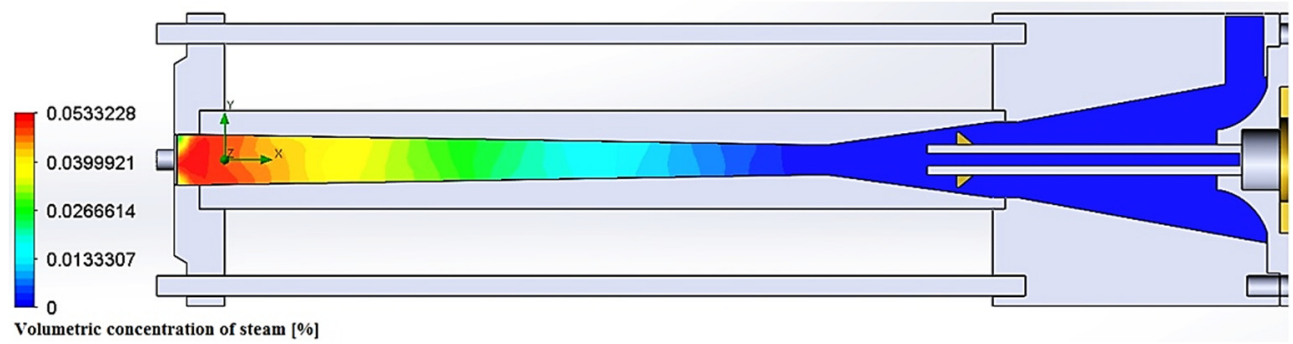

$\mathrm{b}$

Fig. 7. Distribution of vapor volume concentration $\left(\delta_{\mathrm{kp}}=2 \mathrm{~mm}\right)$ : $\mathrm{a}$ - input pressure $0.2 \mathrm{MPa}, \mathrm{b}$ - input pressure $0.4 \mathrm{MPa}$ 
There is also a shift of the cavitation area towards the outlet with input pressure increase (Fig. 8), which confirms the results of experimental and visual studies. Among the studied gaps, the largest volume of the vapor-gas phase is observed for the construction with $\delta_{\text {crit }}=2 \mathrm{~mm}$ (Fig. 8), and the smallest for $\delta_{\text {crit }}=1 \mathrm{~mm}$.

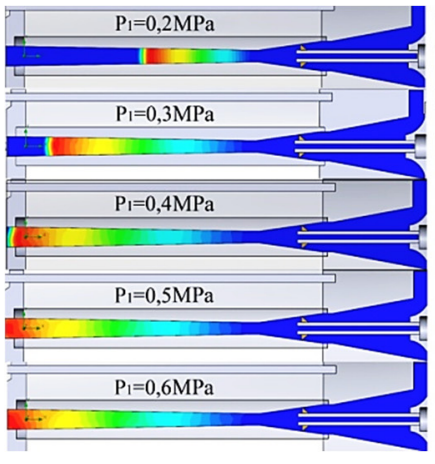

a
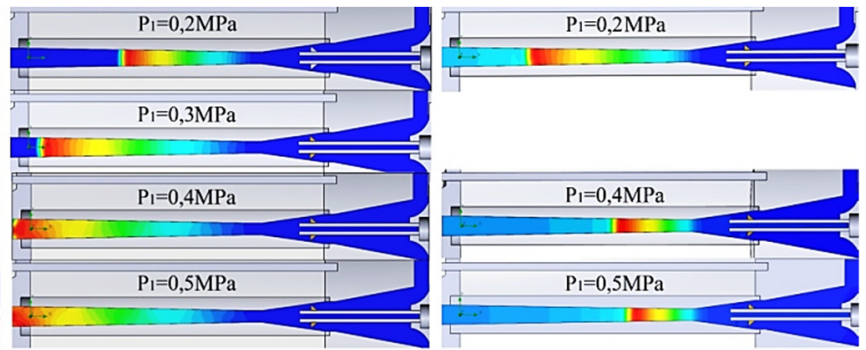

b

Fig. 8. Distribution of volume vapor concentration: $a-\delta_{\text {crit }}=1 \mathrm{~mm} ; \mathrm{b}-\delta_{\text {crit }}=2 \mathrm{~mm} ; \mathrm{c}-\delta_{\text {crit }}=3 \mathrm{~mm}$

The analysis of areas with vapor-gas phase (in Fig. 8) shows that the construction with $\delta_{\text {crit }}=2 \mathrm{~mm}$, with a maximum volume concentration of vapor (Fig. 9), is rational.

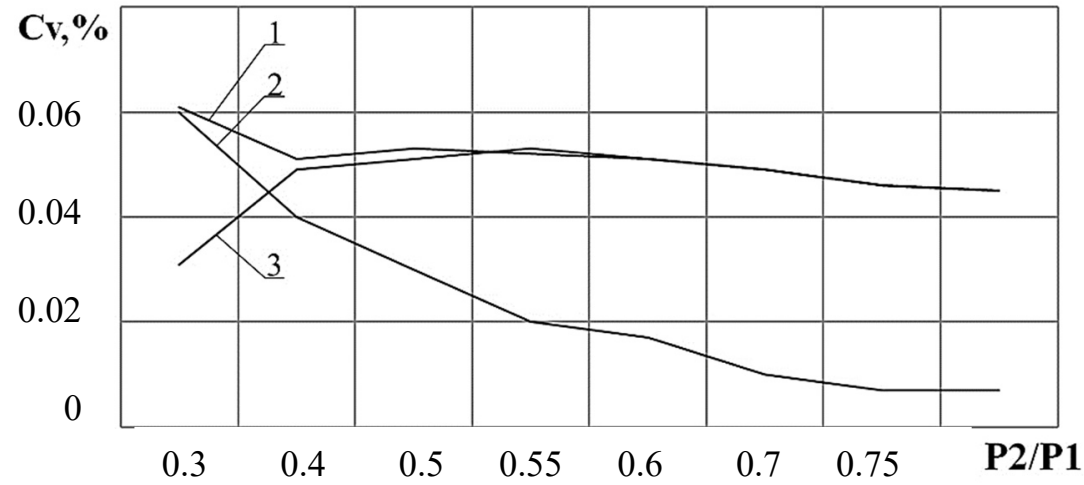

Fig. 9. The dependence of the maximum volume density of the vapor-gas phase on the ratio of pressures $\mathrm{P}_{2} / \mathrm{P}_{1}$ : 1 - gap between the obstacle and the wall $2 \mathrm{~mm}$;

2 - gap between the obstacle and the wall $3 \mathrm{~mm} ; 3$ - the gap between the obstacle and the wall $4 \mathrm{~mm}$

An important parameter that determines the intensity of cavitation impact on the processing medium is the frequency of oscillations. It corresponds to the frequency of separation of cavitation cavities $(f)$ and is determined by the form, geometric and regime parameters of local narrowing. The nature of these frequencies is similar to the frequency of the vortex collapse in the tear-off flow of the cylinder in the normal direction to its axis, which are called Strouhal frequencies of fluid oscillations, and for the hydraulic system with a Venturi tube are defined as the following [10]:

$$
f=S h_{m} \cdot \sqrt{\frac{2 \cdot\left(P_{1}-P_{5}\right)}{\rho_{l i q}}} \cdot \frac{t g \frac{\beta}{2}}{R_{k r}\left(\sqrt{\frac{\mu}{1-\sqrt{1-\tau}}-1}\right)}
$$


where $f$ - pulsation of cavitation bubble;

$S h_{m}$ - the modified Strouhal number;

$P_{l}$ and $P S$ - input pressure within the cavitation area;

$R_{\kappa p}$ - the radii of the critical intersection;

$\mu$ - the cost coefficient;

$\tau$ - the ratio of the outlet pressure to the inlet pressure;

$\beta$ - the angle of the diffuser opening;

$\rho_{\text {liq }}$ - liquid density.

According to the results of studies [11-14] for the implementation of a current characterized by high amplitudes of pressure fluctuations in the pipeline along the Venturi tube, it is necessary that the angle of diffusor opening was $\beta>15^{\circ}$. The most rational angle of diffusior opening is considered to be $\beta=20 \div 30^{\circ}$. In this case, under the condition that the outlet pressure $\mathrm{P}_{2}$ is in the range of $0.1 \div 0.2 \mathrm{MPa}$, the input pressure $-0.12 \ldots 10 \mathrm{MPa}$. Such conditions are fully realized in the device under study.

Experimental dependences of the oscillations frequency $f$ on the parameter $\mathrm{P}_{2} / \mathrm{P}_{1}$ under the pressure $\mathrm{P}_{\text {in }}=0.2-0.5 \mathrm{MPa}$, are shown in Fig. 10. From the figure below, it can be observed that under the change in the ratio $\mathrm{P}_{2} / \mathrm{P}_{1}$ changes the frequency $f$. Thus, with the value of the parameter $\mathrm{P}_{2} / \mathrm{P}_{1}=0.4$, with the increase in the input pressure from 0.2 up to $0.4 \mathrm{MPa}$, the oscillation frequency increases from about 400 up to $750 \mathrm{~Hz}$. The nature of the dependencies is close to the linear one and can be described by the equations shown in Figure 10. The nature of oscillations frequency change significantly dependent on the Reynolds number and input/output pressure change, which is determined by the peculiarities of the design, the characteristics of the pump and determines a certain dependence of the Strouhal criterion on $\operatorname{Re}$ and $\mathrm{P}_{2} / \mathrm{P}_{1}$.

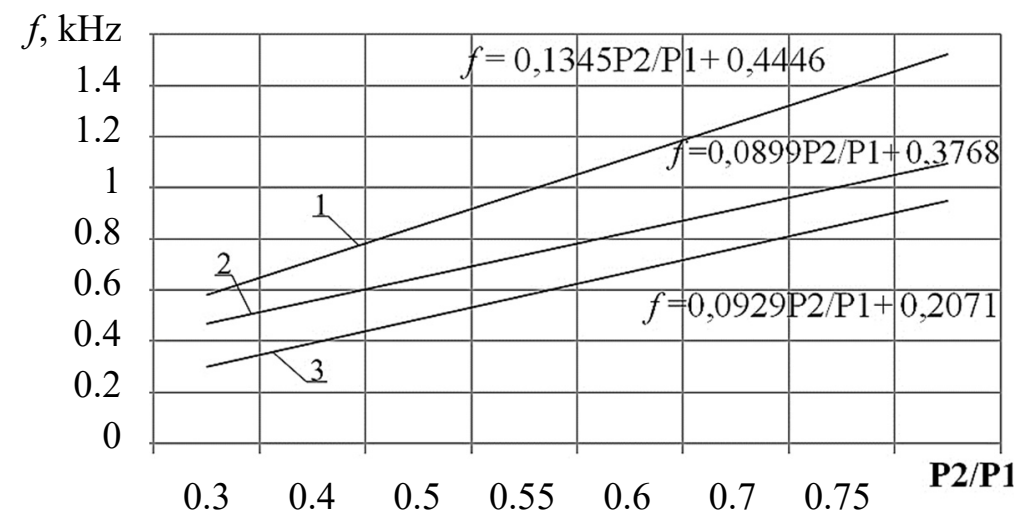

Fig. 10. The dependence of cavity tear-off frequency on pressure difference $\mathrm{P}_{2} / \mathrm{P}_{1}$ : 1 - gap between the obstacle and the wall $2 \mathrm{~mm} ; 2$ - gap between the obstacle and the wall $3 \mathrm{~mm} ; 3$ - the gap between the obstacle and the wall $4 \mathrm{~mm}$

On the other hand, the frequency of oscillations affects the fluid flow, the outlet pressure $\mathrm{P}_{2}$, the hydraulic resistance of the area, which is important for determining the energy consumption and productivity.

The experimental dependences of the $\mathrm{Sh}_{\mathrm{m}}$ change on the dimensionless Reynolds criterion are shown in Fig. 11. 


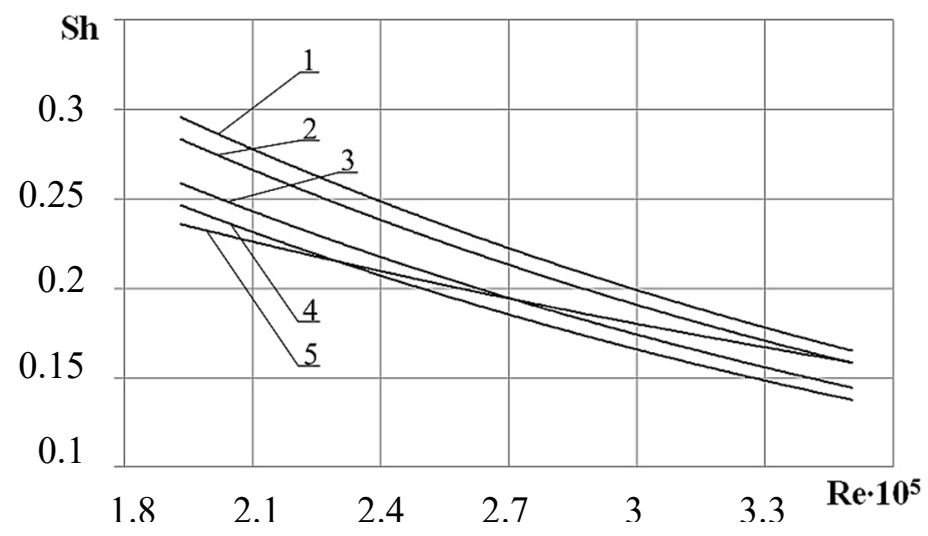

Fig. 11. The dependence of Strouhal number on Reynolds number: 1 - gap between the obstacle and the wall $1 \mathrm{~mm} ; 2$ - gap between the obstacle and the wall $2 \mathrm{~mm} ; 3$ - gap between the obstacle and the wall $3 \mathrm{~mm}$; 4 - gap between the obstacle and the wall $4 \mathrm{~mm} ; 5$ - gap between the obstacle and the wall $5 \mathrm{~mm}$

From the shown results, Strouhal number in the range of Reynolds number $2.2 \cdot 10^{5}-4 \cdot 10^{5}$ is sufficiently stable and decreases with increasing flow velocity in the studied range of velocities. Analyzing the obtained data, it can be assumed that the smaller the diameter of the channel is, the more influence on the flow have hydro-effects near the walls of the working area and the formation of vortices, which contributes to the outlet pressure increase.

In this regard, it is expedient to establish the dependence $\mathrm{Sh}=f\left(\mathrm{Re}, \mathrm{P}_{2} / \mathrm{P}_{1}\right)$. The obtained results provide a practical possibility to determine the nature of this dependence in a certain range of pressure ratios and flow velocity, which ensures the greatest efficiency. Let's consider modes in the range of input pressure from $0.2 \mathrm{MPa}$ up to $0.5 \mathrm{MPa}$. To solve the problem of the complex influence of $\mathrm{Re} ; \mathrm{P}_{2} / \mathrm{P}_{1}$ it is necessary to obtain an interpolation model (regression equation), which determines the influence of these factors on the Strouhal number. Frequency, Reynolds number and pressure difference are selected as independent variables for obtaining this regression equation.

$\mathrm{X}_{1}$ - Reynolds number $(\mathrm{Re})$;

$\mathrm{X}_{2}$ - pressure difference $\left(\mathrm{P}_{2} / \mathrm{P}_{1}\right)$;

The collected statistical information is partially shown in the table.

Table. 1. Input data

\begin{tabular}{|c|c|c|c|c|}
\hline & $\mathbf{X}_{\mathbf{0}}$ & $\mathbf{X}_{\mathbf{1}}$ & $\mathbf{X}_{\mathbf{2}}$ & $\mathbf{Y}$ \\
\hline $\mathbf{1}$ & 1 & 731099.4184 & 0.82 & 0.079442 \\
\hline $\mathbf{2}$ & 1 & 350318.4713 & 0.78 & 0.165792 \\
\hline $\mathbf{3}$ & 1 & 224203.8217 & 0.70 & 0.25905 \\
\hline $\mathbf{4}$ & 1 & 207596.1312 & 0.55 & 0.2798 \\
\hline $\mathbf{5}$ & 1 & 193279.1566 & 0.53 & 0.3005 \\
\hline Sum & 5 & $170649{ }^{\circ} 6.999$ & 3.37 & 1.0846 \\
\hline
\end{tabular}

In order to calculate the coefficients, in this case, a system of normal equations was used which was created on the basis of the least squares method, which provides a sufficiently high accuracy of approximation.

The regression equation for this case has the form:

$$
\mathrm{y}=\mathrm{b}_{0}+\mathrm{b}_{1} \mathrm{X}_{1}+\mathrm{b}_{2} \mathrm{X}_{2}
$$


According to the rule, the system of normal equations gets the form:

$$
\begin{aligned}
& \mathrm{b}_{0} \sum \mathrm{X}_{0}{ }^{2}+\mathrm{b}_{1} \sum \mathrm{X}_{1} \mathrm{X}_{0}+\mathrm{b}_{2} \sum \mathrm{X}_{2} \mathrm{X}_{0}=\sum \mathrm{X}_{0} \mathrm{y} \\
& \mathrm{b}_{0} \sum \mathrm{X}_{0} \mathrm{X}_{1}+\mathrm{b}_{1} \sum \mathrm{X}_{1}{ }^{2}+\mathrm{b}_{2} \sum \mathrm{X}_{2} \mathrm{X}_{1}=\sum \mathrm{X}_{1} \mathrm{y} \\
& \mathrm{b}_{0} \sum \mathrm{X}_{0} \mathrm{X}_{2}+\mathrm{b}_{1} \sum \mathrm{X}_{1} \mathrm{X}_{2}+\mathrm{b}_{2} \sum \mathrm{X}_{2}{ }^{2}=\sum \mathrm{X}_{2} \mathrm{y}
\end{aligned}
$$

In the system of equations we calculate all values under sums based on experimental data. Errors of experiments were determined by the standard equation:

$$
S_{0}^{2}=\frac{1}{N_{0}-1} \sum_{k=1}^{N_{0}}\left(\mathrm{y}_{0 k}-\overline{\mathrm{y}}_{0}\right)^{2}
$$

where $S_{0}$ - mean-root square error;

$N_{0}$ - is the number of parallel experiments;

$y_{0 k}$ - k-th term sequence;

$y_{0}$ - mean forecast error.

The described system of equations was solved according to the standard program. As a result, the numerical values of the coefficients $b_{0}, b_{1}, b_{2}$ were obtained for different conditions.

Table. 2. The coefficients value

\begin{tabular}{|c|c|c|}
\hline $\mathbf{b}_{\mathbf{0}}$ & $\mathbf{b}_{\mathbf{1}}$ & $\mathbf{b}_{\mathbf{2}}$ \\
\hline $1.89 \mathrm{E}-01$ & $1.84 \mathrm{E}-10$ & $3.68 \mathrm{E}-03$ \\
\hline$-5.94 \mathrm{E}-02$ & $7.99 \mathrm{E}-11$ & $1.53 \mathrm{E}-01$ \\
\hline $4.61 \mathrm{E}-02$ & $5.33 \mathrm{E}-10$ & $2.13 \mathrm{E}-01$ \\
\hline $1.54 \mathrm{E}-01$ & $1.48 \mathrm{E}-10$ & $5.85 \mathrm{E}-02$ \\
\hline $9.45 \mathrm{E}-02$ & $2.52 \mathrm{E}-10$ & $1.62 \mathrm{E}-01$ \\
\hline
\end{tabular}

These coefficients vary depending on the three parameters: the input pressure, the gap between the obstacle and the wall, and the cavity pulsation frequency.

The results of the solution of the inverse problem are given in Table 3.

Table. 3. Results of calculations

\begin{tabular}{|c|c|c|c|c|}
\hline$Y_{1}$ experiment & $Y_{2}$ experiment & $Y_{3}$ experiment & $Y_{4}$ experiment & $Y_{5}$ experiment \\
\hline 0.079 & 0.076 & 0.148 & 0069 & 0.066 \\
\hline 0.166 & 0.159 & 0.164 & 0.145 & 0.138 \\
\hline 0.259 & 0.248 & 0.187 & 0.226 & 0.216 \\
\hline 0.280 & 0.268 & 0.220 & 0.245 & 0.233 \\
\hline 0.301 & 0.288 & 0.275 & 0.263 & 0.250 \\
\hline 1.085 & 1.039 & 0.994 & 0.949 & 0.904 \\
\hline Y1 calculation $\mathrm{Y}_{2}$ calculation & $\mathrm{Y}_{3}$ calculation & $\mathrm{Y}_{4}$ calculation & Y $_{5}$ calculation \\
\hline 0.208 & 0.118 & 0.148 & 0.178 & 0.152 \\
\hline 0.211 & 0.147 & 0.164 & 0.182 & 0.161 \\
\hline 0.215 & 0.187 & 0.187 & 0.187 & 0.174 \\
\hline 0.221 & 0.246 & 0.220 & 0.195 & 0.193 \\
\hline 0.231 & 0.342 & 0.275 & 0.208 & 0.224 \\
\hline 1.085 & 1.039 & 0.994 & 0.949 & 0.904 \\
\hline
\end{tabular}

Error of the theoretical calculated results does not exceed $10 \%$. 


\section{Conclusions}

The obtained results of experimental studies of the proposed construction of a device of a hydrodynamic type with a previous input swirling confirmed the formation of a cavitation regime at lower values of flow velocity at the inlet compared to the basic structure without tangential flow input. The dimensionless Reynolds criterion $(\mathrm{Re})$ and gap $(\delta)$ between the wall and the cone were selected as the parameters that determine the volume density of the vapor-gas phase and the size of the cavitation area. It is established that there is a rational regime characterized by a certain ratio of $\mathrm{Re}$ and outlet pressure, under which short cavities with a significant volume of the vapor-gas phase are formed. It is investigated that for the proposed design, the emergence of separate cavitation bubbles occurs when the gap at the entrance to the diffuser $\delta_{\text {crit }}=2 \mathrm{~mm}$. In this case, the critical value of the cavitation number is $\sigma=1.6$. As the flow velocity increases, over time, the film flow that transfers into mixed cavitation mode is observed.

Based on simulation results in the SolidWorks software complex using the Flow simulation module, the results for pressure variations along the working chamber and the vapor-gas phase volume were obtained. Together with the change in pressure, displacement of the cavitation area towards the exit with increasing input pressure was observed, which was confirmed by the results of experimental studies. The analysis of areas with the content of the vapor-gas phase confirmed that the design with the gap $\delta_{\text {crit }}$ $=2 \mathrm{~mm}$, for which there is a maximum volume density of steam, is rational. The obtained dependences of the frequency of oscillations $f$ on the parameter $\mathrm{P}_{2} / \mathrm{P}_{1}$ at the input pressures $\mathrm{P}_{\text {in }}=0.2-0.5 \mathrm{MPa}$, have shown that with the value of the parameter $\mathrm{P}_{2} / \mathrm{P}_{1}=0.4$ with increasing input pressure from 0.2 up to $0,4 \mathrm{MPa}$ the frequency of oscillations increases from 400 to $750 \mathrm{~Hz}$. The character of the obtained mathematical dependences is close to the linear one. It has also been established that the Strouhal number in the Reynolds number range $2.2 \cdot 10^{5}-4 \cdot 10^{5}$ is sufficiently stable and decreases with increasing flow velocity in the studied range of velocities. The comparison of the obtained experimental and theoretical results obtained with respect to the volume of the vapor-gas phase and the dependence of $\mathrm{Sh}=f\left(\mathrm{Re}, \mathrm{P}_{2} / \mathrm{P}_{1}\right)$ confirms their sufficient adequacy (within $10 \%)$.

\section{References}

1. A. A. Dolinsky, G.K. Ivanitsky, The principle of discrete-pulse energy input-new approach to the development of efficient power-saving technologies. Ann. Review of Heat Transfer,Vol. 13, 151-194 (2003).

2. A. A. Dolinsky, G.K. Ivanitsky, Theoretical substantiation of the principle of discrete impulse energy input. Prom. Heat engineering, Vol. 17, 3-28 (1995).

3. A. F Lugovskoy, N.V Chukhraev, Ultrasonic cavitation in modern technologies. Publishing and printing center "Kyiv University", Vol. 13, p. 244 (2007).

4. L. D. Chen, G.M. Faeth, Condensation of Submerged Vapor Jets in Subcooled Liquid. Ibid, Vol. 7, 179-187 (1982).

5. T. M. Vitenko, Ya.M. Humnitsky, Effect of the vapor-gas phase on the kinetics of dissolution under cavitation conditions. East-European magazine for cutting-edge technology, Vol. 4, 4-8 (2009).

6. P. Cerone, J.R. Blake, An ante is the instantaneous streamlines, pathlines and pressure constants for a cavity bubble near the boundary. J. Austral. Math Soc.Ser B, Vol. 8, 4144 (1984). 
7. Y. Tomita, A. Shima, The Machanism of impulsive pressure generation and damage pit formation by bubble collaps. J. Fluid Mach., Vol. 169, 535-564 (1986).

8. Yu. M. Akhmetov, R. R. Kalimullin, V. A. Tselischev, Numerical and physical simulation of fluid flow in a vortex heat generator. Vol. 8, $42-49$ (2010).

9. O. V. Baturin., N. V. Baturin., V.N. Matveev, Calculation of flows of liquids and gases with the help of the universal program complex Fluent. Samara: Publishung agency, Vol. 146, p. 151 (2009).

10. V.V. Pilipenko, Cavitation self-oscillations. Kyiv: Scientific Opinion, Vol. 316, 1-316 (1989).

11. V.V. Pilipenko, I.K. Man'ko, V.A. Zadontsev, Cavitation self-oscillations intensify technological processes. Proceedings of a Fluid Dynamics Panel Workshop, Vol. 4, 321-32-4 (2/1998).

12. V.V. Pilipenko, V.A. Zadontsev, I.K. Man'ko, About high-frequency oscillations in the hydraulic system behind Venturi cavitating tube. Cavitation self-oscillations in pumping systems. Part 2, Naukova Dumka,Vol. 10, 104-113 (1976).

13. V.V. Pilipenko, V.A. Zadontsev, I.K. Man'ko, Yu. Zhulai, N. A. Dzoz, Oscillation generator of fluid pressure. A.c. 1232299 USSR, MKI V06V 1/18, No. 3772504, Vol. 2, p.24-28 (23/05/1986).

14. I. K. Manko, Visual studies of cavitation self-oscillations in a hydraulic system with a transparent Venturi tube. Cavitation self-oscillations in pumping systems. Part 2, Naukova Dumka,Vol. 6, 113-118 (1976). 